\title{
Alignment method for the accurate and precise quantification of tip-surface forces
}

\author{
Daniel Heile $\odot,{ }^{*}$ Reinhard Olbrich $\odot,{ }^{*}$ Michael Reichling $\odot$, and Philipp Rahe $\odot^{\dagger}$ \\ Fachbereich Physik, Universität Osnabrück, Barbarastrasse 7, 49076 Osnabrück, Germany
}

\begin{abstract}
(Received 30 June 2020; revised 14 December 2020; accepted 15 December 2020; published 5 February 2021)
\end{abstract}
\begin{abstract}
We introduce a procedure to determine accurate and precise force-distance curves in dynamic force measurements utilizing a sharp tip. While single force curves are prone to systematic, often unnoticed errors, we present their self-consistent retrieval by an alignment procedure using repetitive measurements with the force probe oscillating at varied amplitude. By processing model data, we show that the procedure provides the valid force curve, the actual oscillation amplitude, and fully compensates thermal drift. The benefit of the method is demonstrated by application to experimental data.
\end{abstract}

DOI: 10.1103/PhysRevB.103.075409

\section{INTRODUCTION}

The understanding of binding in matter is a great success of quantum mechanics and over a century, elaborate theories describing the interaction between atoms and molecules by electromagnetic forces have been developed [1]. While, for systems up to a certain size, chemical bonds and physical interactions can precisely be described by the interaction between individual atoms, this is not practical and elucidating for the description of binding between mesoscopic and macroscopic bodies. For such objects, binding is commonly discussed in the framework of integral physical interactions phenomenologically described as adhesion [2]. Most relevant in nanoscale force measurements, there is no strict border between these two types of descriptions.

The measurement of forces between atoms, molecules, and nanoscale objects has been pushed to the physical limits [3-5] and is of great interest in diverse fields of science and technology including high-resolution analysis of inorganic [6-8], organic [9-11], and biological surfaces [12-14], three-dimensional (3D) force mapping [15-18], Casimir force measurements [19], nanomechanical material characterization [20-24], capillary force studies [25-29], hydration layer analysis [30-33], the study of atom-specific reactivity [34,35], mechanochemistry [36-38], quantum dot microscopy [39], and single-spin detection $[40,41]$. The technique of noncontact atomic force microscopy (NC-AFM) allows dynamic force measurements between an oscillating sharp tip and a surface of any kind, with a resolution down to the atomic scale [42-45]. Still, the interpretation of force measurements at the nanoscale is most difficult as this is the crossover regime where forces between individual atoms and molecules as well as forces between mesoscopic bodies act together. In this regime, the force-distance law is not a simple function of the

\footnotetext{
${ }^{*}$ These authors contributed equally to this work.

†prahe@uni-osnabrueck.de
}

Published by the American Physical Society under the terms of the Creative Commons Attribution 4.0 International license. Further distribution of this work must maintain attribution to the author(s) and the published article's title, journal citation, and DOI. body separation, but is often described as a sum of contributions based on different models [46]. Utmost precision in measuring and analyzing a force-distance curve is required to test models and to quantify the nanoscale interactions.

The conceptual difficulties in describing tip-surface forces have to be seen alongside experimental peculiarities severely limiting accurate dynamic force measurements. First, the primary measurement signal in NC-AFM is not the force, but the shift in the resonance frequency of a high- $Q$ force probe oscillating in the force field above the surface [44]. The force curve is derived from frequency shift data by numerical deconvolution, commonly referred to as force inversion $[47,48]$. The result of this inversion can be ill-posed [49], can exhibit large errors in a range of relevant amplitudes [50], or can include severely amplified detection system noise [51]. Second, the force inversion algorithms yield valid results only if the oscillation amplitude is known accurately $[48,50]$. Third, strong gradients in the force field make the measurement susceptible to minute deviations of the probe trajectory from the target curve as they result, for example, from thermal drift even when applying active drift compensation [52,53].

In the present paper, we introduce the force curve alignment (FCA) method facilitating the accurate and precise measurement of force curves by circumventing experimental peculiarities and ultimately removing systematic errors in widely accepted present practice. The central concept is the repetitive measurement of the tip-surface interaction with different probe oscillation amplitudes, delivering a data set, which is robust against disturbing impacts. While the presence of systematic errors can hardly be identified from a single interaction curve, a match of the individual force curves by an optimisation algorithm enables a self-consistent determination of the error-free tip-surface interaction force curve. A key challenge for retrieving the force from frequency shift data is accurately determining the probe oscillation amplitude $A$. The physical oscillation amplitude $A$ is derived from the measured voltage amplitude $V_{A}$ via the calibration factor $S$ defined as $A=S \cdot V_{A}$. The calibration is principally straightforward, however, obtaining an accurate result is practically most difficult. Among various methods suggested [54-61], those involving the tip-surface interaction $[55,56,58]$ ensure that the 

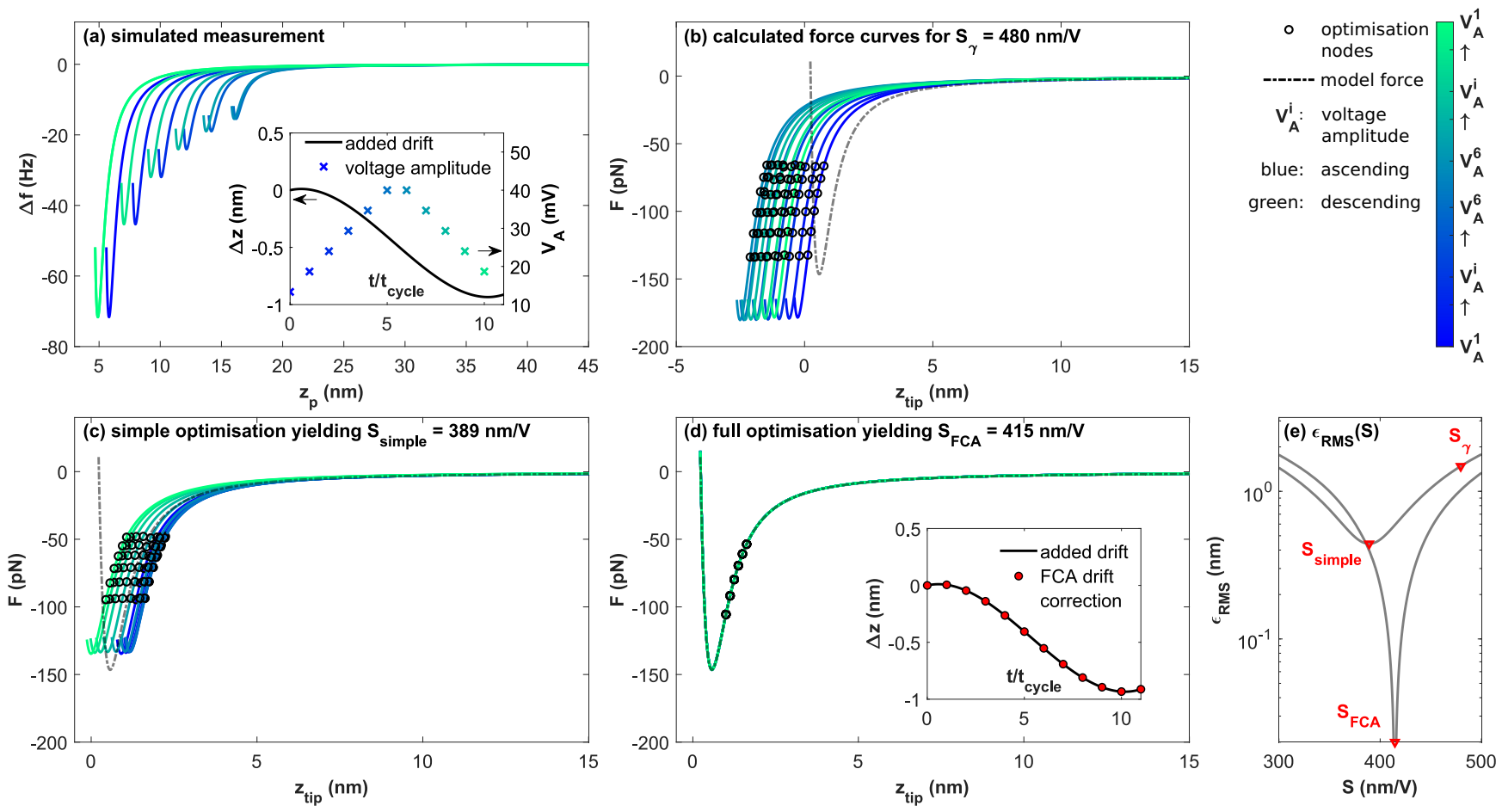

FIG. 1. Stages of model data processing with the FCA method. (a) Simulated frequency shift $\Delta f^{i}\left[z_{\mathrm{p}}+\Delta z\left(t^{i}\right)\right]$ data for six ascending and six descending voltage amplitudes $V_{A}^{i}$ (see inset) including thermal drift $\Delta z(t)$ (see inset). $z_{\mathrm{p}}$ denotes the piezo position. (b) Curves $F^{i}\left(z_{\text {tip }}\right)$ of the normal force as a function of the tip position $z_{\text {tip }}$ derived from the $\Delta f^{i}\left[z_{\mathrm{p}}+\Delta z\left(t^{i}\right)\right]$ data using the starting value $S_{\gamma}=480 \mathrm{~nm} / \mathrm{V}$, zero drift, and the Sader-Jarvis force inversion algorithm [48]. $z_{\text {tip }}$ is defined as the minimum tip-surface distance attained in the lower turning point of the oscillatory tip motion (see Fig. 4 in Appendix A). The dash-dotted line is the model force curve used for generating the artificial frequency shift data. Black circles are optimization nodes used for the FCA correction. (c) Simplified FCA alignment (without considering thermal drift) yielding $S_{\text {simple }}=389 \mathrm{~nm} / \mathrm{V}$. (d) Accurate force curves resulting from applying the full FCA procedure yielding $S_{\mathrm{FCA}}=415 \mathrm{~nm} / \mathrm{V}$. The inset demonstrates the excellent match between the $\Delta z\left(t^{i}\right)$ corrections resulting from FCA and the artificial $\Delta z(t)$ input. (e) Residual error $\epsilon_{\mathrm{RMS}}$ as a function of $S$ together with the positions of the approximations $S_{\gamma}$ and $S_{\text {simple }}$ as well as the accurate value $S_{\mathrm{FCA}}$.

calibrated amplitude corresponds to the position of the tip. This is a most important aspect which challenges calibration methods based on interferometry: Although the latter methods yield in principle very precise distance measurements as they use the wavelength or speed of light as a reference of unprecedented accuracy [54,59], a slight misalignment of the laser spot on the cantilever will introduce a systematic error with respect to the tip amplitude relevant for the force measurement. Conceptually advantageous is the $\gamma$-method [55], which is based on an approximation by the normalized frequency shift [62]. As demonstrated in Appendix B, this method may yield reasonably good results under certain conditions, but may also fail and is, therefore, not suitable when seeking for a highly accurate calibration. The limitations of the presently available amplitude calibration procedures have been a major driving force for developing the FCA method that inherently yields an accurate value for $S$. In the following, first the validity of the FCA method is demonstrated by applying FCA to model data that are based on measured force curves. Second, the practicability of FCA is shown by application to experimental data acquired on a $\mathrm{CeO}_{2}(111)$ surface decorated with gold nanoparticles.

\section{FORCE CURVE ALIGNMENT METHOD}

Intermediate and final results of a model force measurement run are compiled in Fig. 1 for demonstration and verification of the FCA method. FCA relies on the measurement of a set of $2 N$ (here: $N=6$ ) tip retraction and approach cycles, with ascending (blue shadings) and descending (green shadings) amplitude voltage values $V_{A}^{i}$. A set of the corresponding artificial frequency shift $\Delta f^{i}\left(z_{\mathrm{p}}\right)$ curves [Fig. 1(a)] is calculated from a model force curve $F_{\text {mod }}\left(z_{\text {tip }}\right)$ resembling an experimental measurement (see Appendix A) by applying the appropriate transformation [44] for the set of voltage amplitudes $V_{A}^{i}$ [see inset in Fig. 1(a)] with $S=415 \mathrm{~nm} / \mathrm{V}$. Furthermore, artificial nonlinear thermal drift $\Delta z(t)$ [see inset in Fig. 1(a)] is added to the tip positions $z_{\text {tip }}$ with magnitudes and velocities of the drift chosen to match values typically found in experiments. As a result, the drift-influenced data of a typical experiment is generated. While the resulting $\Delta f^{i}\left(z_{\mathrm{p}}\right)$ curves representing different $V_{A}^{i}$ as shown in Fig. 1(a) naturally differ from each other due to the amplitude-dependency of the absolute frequency-shift value [62], curves having the same $V_{A}^{i}$ value differ due to the introduced thermal drift $\Delta z\left(t^{i}\right)$.

An estimate for the amplitude calibration factor and thermal drift parameters is used to calculate the corresponding set of force curves as the starting point for the FCA optimization. We choose $S_{\gamma}=480 \mathrm{~nm} / \mathrm{V}$ as the estimate from the $\gamma$-method [55] (see Appendix B) and start with zero drift. The set of resulting force curves $F^{i}\left(z_{\text {tip }}\right)$ calculated with the Sader-Jarvis algorithm [48] is shown in Fig. 1(b). It is immediately apparent that all $F^{i}\left(z_{\text {tip }}\right)$ curves are shifted along the $z_{\text {tip }}$ 
axis relative to each other, differ in their slope and curvature, and none of them coincides with the true force curve that is known for this simulation and displayed as a dash-dotted line in Figs. 1(b)-1(d). Similar results are obtained with the matrix algorithm [47] (see Appendix H).

In a simple version of the FCA optimisation, force curves $F^{i}\left(z_{\text {tip }}\right)$ are corrected for the error in $S$ but not for thermal drift. To this end, six optimization nodes are defined on each curve [black circles in Figs. 1(b)-1(d)], each belonging to a set of six given force values along the curve. With one free parameter, namely $S$, the optimization procedure recalculates the curves with iteratively optimized $S$ so that the value for $\epsilon_{\mathrm{RMS}}$ representing the RMS value of the deviation between the optimization nodes of each curve from a chosen reference curve (the first curve in the data set) is minimized. The improvement by the optimization is evident from Fig. 1(c) showing force curves with a reduced spread close to the true curve. This optimization yields a better estimate for the amplitude calibration factor of $S_{\text {simple }}=389 \mathrm{~nm} / \mathrm{V}$, however, the residual spread of the force curves is evidence for their distortion by thermal drift.

To retrieve the true force curve, we apply the full FCA optimization procedure including the adjustment of a third-order drift polynomial for approximating the thermal drift $\Delta z(t)$ to the $F^{i}\left(z_{\text {tip }}\right)$ curves from Fig. 1(b) with results shown in Fig. 1(d) (see Appendixes E and F for the measurement and analysis protocol, respectively, and the Supplemental Material [63] for the program code). The full optimization yields $12 F_{\mathrm{FCA}}^{i}\left(z_{\text {tip }}\right)$ force curves not discernible from each other and perfectly matching the true force curve. In addition to fully recovering the artificially obscured force data, the FCA method yields the correct amplitude calibration factor $S_{\mathrm{FCA}}=$ $415 \mathrm{~nm} / \mathrm{V}$. The inset in Fig. 1(d) presents an excellent match between the discrete drift corrections determined by the FCA optimization and the artificially introduced drift polynomial. The graph shown in Fig. 1(e) demonstrates the reliability of the FCA method when applied to a perfect set of data: for the full FCA optimisation, the function $\epsilon_{\mathrm{RMS}}(S)$ describing the residual error exhibits one sharp minimum allowing for a precise determination of $S$ and the true force curve. Note that the value of $\epsilon_{\mathrm{RMS}}$ corresponding to $S_{\mathrm{FCA}}$ is nearly ten orders of magnitudes smaller than it is drawn in Fig. 1(e) and solely represents the numerical error.

\section{RESULTS AND DISCUSSION}

The practicability of the method is demonstrated for the real experimental data shown in Fig. 2. We performed measurements on a well-prepared $\mathrm{CeO}_{2}(111)$ surface exhibiting large flat terraces separated by steps with the height of a O-Ce-O triple layer [64-66]. Regularly shaped gold clusters with lateral dimensions of typically $10 \mathrm{~nm}$ and measured heights of typically 1 to $3 \mathrm{~nm}$ were introduced as nanoscale irregularities. Two strongly different tips were used to acquire interaction curves representative for two typical experimental conditions in dynamic force measurements at room temperature under ultra-high vacuum conditions (see Appendix D for details). The first tip was a silicon tip subjected to sputtering and potential contamination after exposure to the residual gas and sample contact. This bare silicon tip acts as a representative for "sharp" tips typically used for NC-AFM imaging.
The second tip was a Pt/Ir-coated silicon tip as typically used for Kelvin probe force microscopy with a usually larger tip radius due to the coating. An NC-AFM image of the surface taken with a silicon tip is shown in the inset of Fig. 2(a). The two red dots mark typical positions where force curves were acquired: Central on a single gold cluster ("on-cluster") and in a region representing the flat surface ("off-cluster"). Care has been taken in the latter case to measure forces far away from clusters, step edges, or other disturbances.

In full analogy to the analysis steps applied to the simulated data, the graphs in Fig. 2(a) show the original measurements $\Delta f^{i}\left(z_{\mathrm{p}}\right)$ taken with the bare silicon tip. At both positions, the $\Delta f^{i}\left(z_{\mathrm{p}}\right)$ curves were sampled in 12 approach/retract cycles with ascending and descending voltage amplitudes $V_{A}^{i}$ ranging from 13.3 to $40 \mathrm{mV}$. Note that data were deliberately not acquired close to or beyond the minima of the force curves to avoid any tip change that would severely compromise the force measurement. The graphs in Fig. 2(b) show the $F^{i}\left(z_{\text {tip }}\right)$ data after force inversion but before the optimization. We used a starting value of $S=S_{\gamma}=480 \mathrm{~nm} / \mathrm{V}$ and assumed zero drift for the start of on-cluster and off-cluster experiments.

Figure 2(c) represents the result using the full FCA procedure for this series of measurements. Individual data points from the aligned force curves are shown as gray dots while average curves determined for off-cluster and on-cluster measurements are shown as green and orange lines, respectively. It is immediately apparent that the off-cluster measurement yields an excellent match of the aligned forces and unambiguously provides the tip-sample force curve. The optimization yields $S=S_{\mathrm{FCA}}^{\text {off }}=(414.6 \pm 1.9) \mathrm{nm} / \mathrm{V}$ as the accurate result for this measurement. The specified error reflects the accuracy of determining the minimum in $\epsilon_{\mathrm{RMS}}$ as estimated from the width of the $\epsilon_{\mathrm{RMS}}(S)$ curve (see Appendix G for details).

The spread across the individual force curves is larger in the on-cluster data shown in Fig. 2(c), in agreement with observing a larger spread in the $\Delta z\left(t^{i}\right)$ values (orange line in the left inset) and an $\epsilon_{\mathrm{RMS}}\left(S_{\mathrm{FCA}}^{\text {on }}\right)$ value larger by a factor of 4.4 than $\epsilon_{\mathrm{RMS}}\left(S_{\mathrm{FCA}}^{\text {off }}\right)$ (right inset). The amplitude calibration factor of $S_{\mathrm{FCA}}^{\text {on }}=(383 \pm 8) \mathrm{nm} / \mathrm{V}$ is furthermore smaller than $S_{\mathrm{FCA}}^{\text {off }}$. We attribute these differences to a systematic error introduced by the inclination of the direction of probe oscillation from the surface normal ( $\alpha=12.5^{\circ}$ for our instrument). The inclination is not compatible with the force inversion algorithm integrating the weighted frequency shift along the surface normal (see Appendix $\mathrm{C}$ for a discussion). On a homogeneous and isotropic surface, where the force is a function of $z_{\text {tip }}$ only, the inclination can be compensated by a projection of the oscillation coordinate to the surface normal. However, when measuring above a cluster with dimensions comparable to the tip radius, the tip experiences lateral force gradients and measurements with different oscillation amplitude will be differently influenced by them, ultimately resulting in incorrect force measurements. This is convincing evidence that the FCA method is able to unravel so far unnoticed sources for inaccurate force measurements.

Measurements with the Pt/Ir-coated tip were performed and analyzed in exactly the same manner with final results for one representative measurement compiled in Fig. 2(d). For this probe we find values of $S_{\mathrm{FCA}}^{\mathrm{on}}=(165.5 \pm 0.9) \mathrm{nm} / \mathrm{V}$ and $S_{\mathrm{FCA}}^{\text {off }}=(169.3 \pm 0.5) \mathrm{nm} / \mathrm{V}$, both significantly smaller than the value for the silicon tip due to the high optical reflectivity 

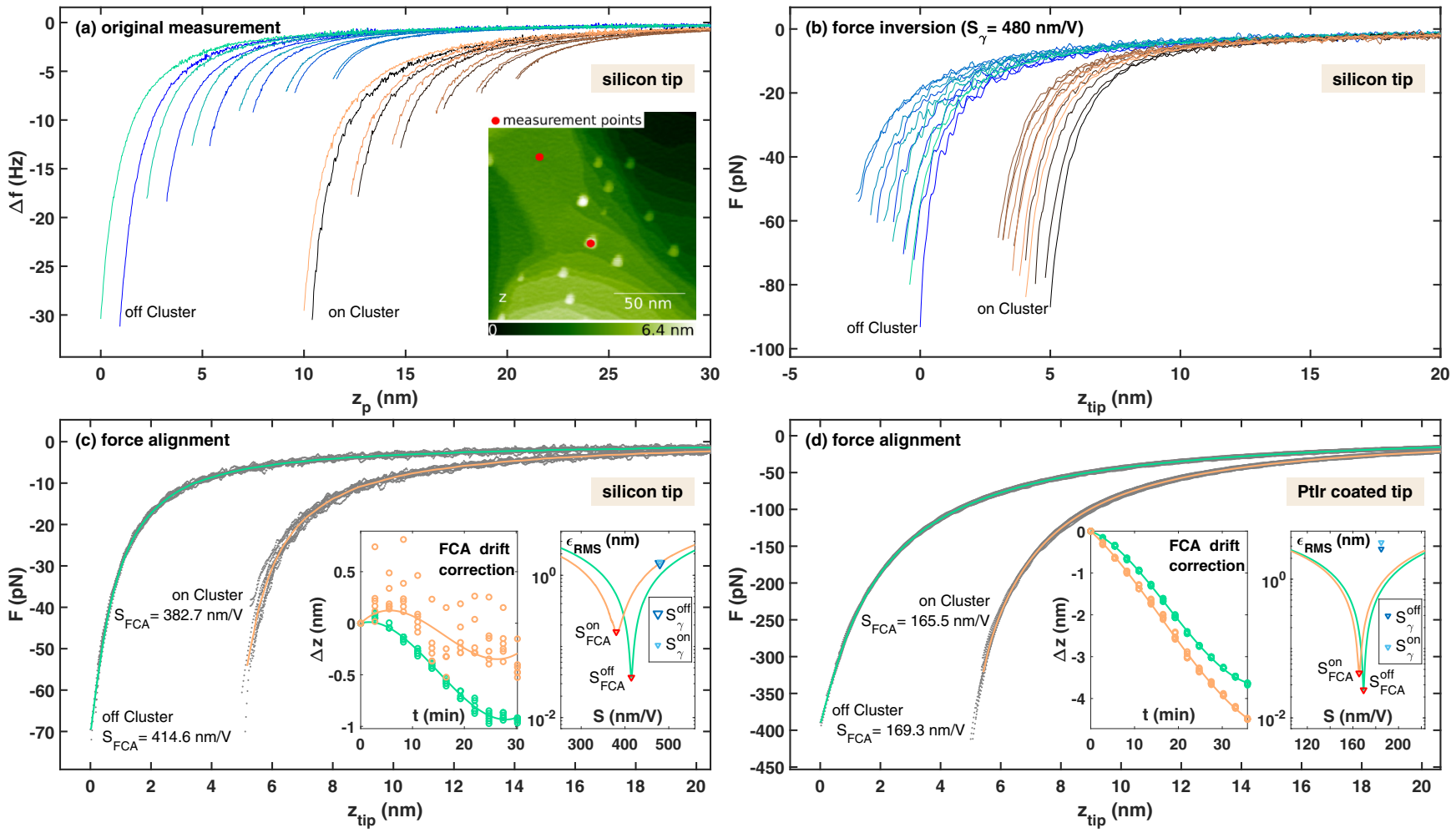

FIG. 2. Experimental force measurements on a $\mathrm{CeO}_{2}$ (111) surface decorated with gold clusters. Results compiled in (a)-(c) represent measurements taken with the bare silicon tip while panel (d) displays the final results of measurements performed with a Pt/Ir coated silicon tip. The steps of force inversion and full FCA optimisation were carried out in exactly the same manner as for the model data analysis shown in Fig. 1. Measurements were performed for positions at the center of gold clusters (on-cluster) as well as on atomically flat terraces far from step edges and other disturbances (off-cluster) and are coded with blue/green and black/orange colors, respectively. On-cluster and off-cluster positions are exemplary shown as red dots in the inset of (a), which is a NC-AFM topography image of the gold decorated $\mathrm{CeO}_{2}(111)$ surface taken with the bare silicon tip. The green and orange curves shown in (c) and (d) are averaged curves of the force curve data plotted as gray points. The left insets in (c) and (d) display the drift polynomial $\Delta z(t)$ yielded by the FCA optimisation (solid line) and the deviations from the mean curve found for the six optimisation nodes (circles). The right insets in (c) and (d) display $\epsilon_{\mathrm{RMS}}(S)$, similar to Fig. 1(e), for on- and off-cluster measurements.

of the metal coated cantilever. Other basic observations are the very same as for the silicon tip, yet, the more slowly decaying forces highlight the larger tip radius resulting from the $\mathrm{Pt} / \mathrm{Ir}$ coating. The difference between $S_{\mathrm{FCA}}^{\text {on }}$ und $S_{\mathrm{FCA}}^{\text {off }}$ is tiny compared to the bare silicon tip measurement. This is intelligible considering that a tip with a larger tip radius experiences smaller lateral force gradients as the force is averaged over a larger area.

\section{CONCLUSIONS}

The measurements and their analysis in the framework of FCA deliver a very consistent picture. For a set of high quality data, the FCA method yields the true tip-surface interaction force curve free from critical systematic errors as well as an accurate and highly precise value for the amplitude calibration factor. It is noteworthy that an accurate value for $S$ is most important for an accurate determination of the modal sensor stiffness $k_{0}$ [67], another key parameter for force measurements. The FCA method can easily be adapted to the needs of the experiment, specifically, the user can tailor the FCA optimization function. If a measurement is compromised by any kind of disturbance that cannot be compensated for by the user implemented optimization function, force curves will not coincide what clearly indicates a problem with the dataset or the chosen correction function. FCA offers great flexibility in its application as it is compatible with a direct force measurement as well as with any force inversion algorithm. This especially concerns force mapping experiments, where thermal drift that is usually the major problem for long-lasting measurements, is fully compensated by FCA. Hence, FCA can equally well be used for static AFM, intermitted contact AFM, and NC-AFM. It facilitates the quantitative analysis of force measurements with unprecedented accuracy and precision in any environment and at any temperature provided the force curve is deterministic what implies sufficient stability of tip and surface. It can be anticipated that FCA will have a significant impact in a large number of fields in physics, chemistry, and biology as well as in cross-disciplinary sciences and nanotechnology.

\section{ACKNOWLEDGMENTS}

This work was supported by the Deutsche Forschungsgemeinschaft via Grants No. RA2832/1-1, No. RE1186/21-1, and No. RE1186/23-1. We thank Jonas Heggemann for most helpful discussions on the method. 

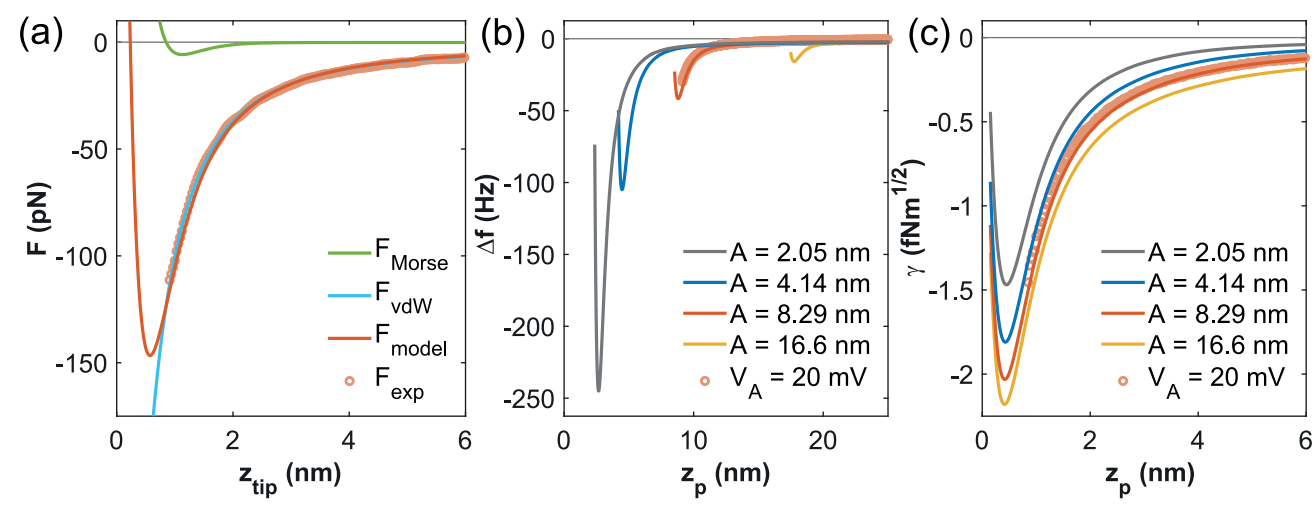

FIG. 3. (a) Interaction force $F$ used for the simulations. A combined Morse (green line) and van der Waals (blue line) interaction [69] is adjusted to fit an experimentally measured force-distance curve (circles). (b) Frequency shift $\Delta f$ and (c) normalized frequency shift $\gamma$ calculated for different amplitudes from the force in (a). Experimental data included as circles.

\section{APPENDIX A: MODEL FORCE CURVES}

To create a model force curve for testing the FCA method, we use a combination of Morse and van der Waals interaction forces for all simulations

$$
F_{\text {model }}\left(z_{\mathrm{ts}}\right)=F_{\text {Morse }}\left(z_{\mathrm{ts}}\right)+F_{\mathrm{vdW}}\left(z_{\mathrm{ts}}\right),
$$

similar to the separation of interactions in Refs. [46,68]. One contribution is the force corresponding to the Morse potential

$$
V_{\text {Morse }}\left(z_{\mathrm{ts}}\right)=E_{b}\left[2 e^{-\kappa\left(z_{\mathrm{ts}}-\sigma_{0}\right)}-e^{-2 \kappa\left(z_{\mathrm{ts}}-\sigma_{0}\right)}\right],
$$

describing the short-range interaction by

$$
F_{\text {Morse }}\left(z_{\mathrm{ts}}\right)=2 E_{b} \kappa\left[-e^{-\kappa\left(z_{\mathrm{ts}}-\sigma_{0}\right)}+e^{-2 \kappa\left(z_{\mathrm{ts}}-\sigma_{0}\right)}\right] .
$$

The van der Waals force derived by Argento and French [69] as the force between a cone with half opening angle $\Theta$, terminated with a half sphere of radius $R$, and Hamaker constant $H$

$$
\begin{aligned}
F_{\mathrm{vdW}}\left(z_{\mathrm{ts}}\right)= & -\frac{H R^{2}(\sin \Theta-1)[(R-\hat{z}) \sin \Theta-R-\hat{z}]}{6 \hat{z}^{2}(R+\hat{z}-R \sin \Theta)^{2}} \\
& -\frac{H \tan \Theta[(\hat{z}+R) \sin \Theta+R \cos (2 \Theta)]}{6 \cos (\Theta)(\hat{z}+R-R \sin \Theta)^{2}}
\end{aligned}
$$

is used to describe the interaction of the mesoscopic tip with the surface. Here, $\hat{z}=z_{\text {ts }}+z_{\text {offset }}$, where $z_{\text {offset }}$ describes the height of the nanotip attached to the mesoscopic tip. Accordingly, we find for the force gradients

$$
\begin{aligned}
& k_{\text {Morse }}\left(z_{\mathrm{ts}}\right)=2 E_{b} \kappa^{2}\left[e^{-\kappa\left(z_{\mathrm{ts}}-\sigma_{0}\right)}-2 e^{-2 \kappa\left(z_{\mathrm{ts}}-\sigma_{0}\right)}\right] \\
& k_{\mathrm{vdW}}\left(z_{\mathrm{ts}}\right)= \frac{H}{6 \hat{z}^{3}(R+\hat{z}-R \sin \Theta)^{2}} \\
& \times\left[R \left\{2 R^{2}+3 R \hat{z}+(2 R-\hat{z})\right.\right. \\
&\left.\times(-2(R+\hat{z})+R \sin (\Theta)) \sin \Theta\}+\hat{z}^{3} \tan ^{2} \Theta\right] .
\end{aligned}
$$

These terms are used within the quantitative AFM framework [44] to calculate the frequency shift curve $\Delta f\left(z_{\mathrm{p}}\right)$. The starting point is an experimentally obtained force curve shown as orange circles in Fig. 3(a). The parameters $H, \Theta$, and $z_{\text {offset }}$ of the van der Waals interaction were fitted (with $R=5 \mathrm{~nm}$ kept fixed) to these experimental data acquired on a $\mathrm{CeO}_{2}(111)$ surface with a bare silicon tip. For the Morse potential we adapted a model for the interaction between $\mathrm{Si}$ atoms [50] with the herein used parameters specified in Table I.

Fiure $3(\mathrm{a})$ presents the experimental force data $\left(F_{\text {exp }}\right.$, circles) as well as the calculated Morse ( $F_{\text {Morse }}$, green), van der Waals $\left(F_{\mathrm{vdW}}\right.$, blue $)$, and total $\left(F_{\text {model }}\right.$, red) force curves. The latter curves are calculated using the parameters listed in Table I.

The model force data $F_{\text {model }}\left(z_{\text {tip }}\right)$ is used to calculate frequency shift $\Delta f\left(z_{\mathrm{p}}\right)$ curves for representative oscillation amplitude ranging from about 2 to $20 \mathrm{~nm}$ [Fig. 3(b)] with the quantitative AFM framework [44]. Experimental $\Delta f$ data are included in Fig. 3(b) and match the simulation result for $A=8.5 \mathrm{~nm}$ as expected from the sensitivity factor and the chosen voltage amplitude. In a further step, $\Delta f\left(z_{\mathrm{p}}\right)$ curves are converted into $\gamma\left(z_{\mathrm{p}}\right)$ curves using the definition of the normalised frequency shift [62] [Fig. 3(c)]. This figure highlights the limitations of the concept of the normalized frequency shift: even for the two largest amplitudes of 8.29 and $16.6 \mathrm{~nm}$, the $\gamma\left(z_{\mathrm{p}}\right)$ curves do not match.

In Fig. 4 we explain the use of the symbols to describe the probe position along $z$. Once the coarse approach is completed, the tip is positioned by adjusting the piezoposition $z_{\mathrm{p}}$ to yield a certain tip-sample distance $z_{\mathrm{ts}}$. While the relative movement in $z_{\mathrm{p}}$ is well known from a respective calibration, the absolute position $z_{\text {ts }}$ is a priori not known. As the force curve $F\left(z_{\mathrm{ts}}\right)$ does not have a well-defined endpoint, the origin $z=0$ cannot easily be defined and is practically chosen with an arbitrary offset. In a dynamic measurement, $z_{\text {ts }}$ is a function

TABLE I. Parameters for the Morse and van der Waals interactions used within the simulations.

\begin{tabular}{lc}
\hline \hline Morse interaction & \\
$E_{\mathrm{b}}$ & $4.638 \mathrm{zJ}$ \\
$\sigma_{0}$ & $850 \mathrm{pm}$ \\
$\kappa$ & $2.50 \mathrm{~nm}^{-1}$ \\
van der Waals interaction & \\
$H$ & $357.619 \mathrm{zJ}$ \\
$\Theta$ & $29.7^{\circ}$ \\
$R$ & $5 \mathrm{~nm}$ \\
$z_{\text {offset }}$ & $583.04 \mathrm{pm}$ \\
\hline
\end{tabular}




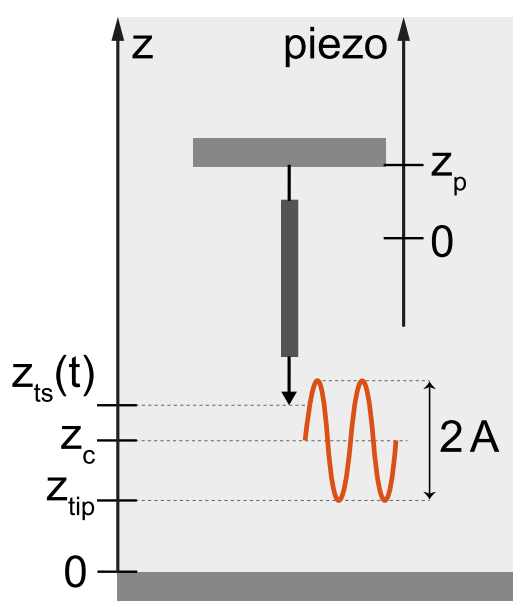

FIG. 4. Axis system describing the vertical $z$ positions. The notation follows Ref. [44].

of time and $z_{\mathrm{ts}}(t)$ is often assumed as a quasiharmonic oscillation around the center position $z_{\mathrm{c}}$ with amplitude $A$ [44]. The Sader-Jarvis force inversion algorithm is implemented to yield the force as a function of the tip-surface distance specified by the lower turning point $z_{\text {tip }}$ of the oscillation. Therefore, we plot measured frequency shift curves $\Delta f\left(z_{\mathrm{p}}\right)$ as a function of the known coordinate $z_{\mathrm{p}}$ with an arbitrarily chosen origin. Likewise, the force curve $F\left(z_{\text {tip }}\right)$ determined by the force inversion is plotted as a function of the coordinate $z_{\text {tip }}$ with an arbitrarily chosen origin.

\section{APPENDIX B: $\gamma$-METHOD}

The $\gamma$-method is a well-established standard procedure to determine the sensitivity factor $S$ based on a sequence of approach and retract cycles performed with a set of voltage amplitudes $V_{\mathrm{A}}^{i}$. These voltage amplitudes represent a priori unknown physical oscillation amplitudes $A^{i}$ [55]. For each set point representing a certain $V_{\mathrm{A}}^{i}$, the mean tip-sample distance $z_{c}=\overline{z_{t s}}$ is adjusted by changing the piezo position $z_{\mathrm{p}}^{i}$ to a value representing the same predefined tip-sample interaction. Crucially, the tip-sample interaction cannot be measured but is commonly estimated by the normalised frequency shift $\gamma=k_{0} A^{3 / 2} \Delta f / f_{0}$ where $k_{0}$ is the modal probe stiffness, $\Delta f$ the observed frequency shift, and $f_{0}$ the probe eigenfrequency [62].

In the calibration experiment, $V_{\mathrm{A}}$ is stepwise increased and decreased (up and down), while the given normalized frequency shift $\gamma_{\text {set }}$ is kept constant by the choice of an appropriate frequency-shift setpoint $\Delta f_{\text {set }}^{i}$. As a consequence, the topography feedback loop adjusts $z_{\mathrm{p}}^{i}$ accordingly. The amplitude calibration factor $S_{\gamma, z}=\Delta z_{\mathrm{p}} / \Delta V_{A}$ is the slope of the graph in a plot of the piezopositions $z_{\mathrm{p}}^{i}$ as a function of the voltage amplitudes $V_{A}^{i}$. An exemplary result of this procedure for a silicon tip over the ceria surface is shown in Fig. 5. Note that in this and all other measurements, the $\gamma$-method does not directly yield $S_{\gamma}$, but $S_{\gamma, z}=S_{\gamma} \cos (\alpha)$ as the direction of the oscillation is usually inclined by an angle of $\alpha$ (in the present setup $\alpha \approx 12.5^{\circ}$ ) with respect to the surface normal (see Appendix C for further details). The $S_{\gamma}=S_{\gamma, z} / \cos \alpha$ value derived from this calibration curve was used as the

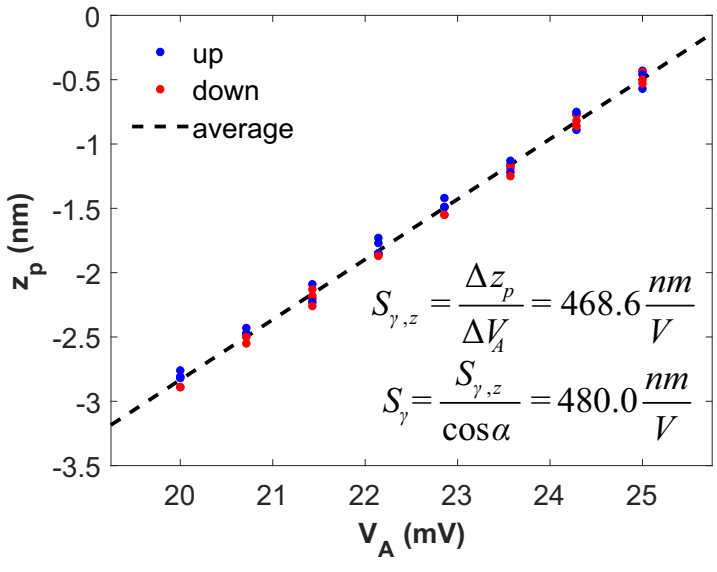

FIG. 5. Determination of the sensitivity factor $S$ utilizing the $\gamma$-method for a silicon probe over a ceria surface. $S_{\gamma, z}$ follows from the slope of a linear regression and $S_{\gamma}$ from a correction for the inclination $\alpha$ between the cantilever oscillation and the surface normal ( $\alpha \approx 12.5^{\circ}$ for the herein used system).

starting value for the force curve alignment in Fig. 1(b) of the main paper.

The accuracy of the $\gamma$-method is analyzed by simulations, yielding results shown in Fig. 6 together with corresponding experimental measurements. The realistic model force curve as described in Appendix $\mathrm{A}$ is used for calculating $\gamma(z)$ curves for different $V_{\mathrm{A}}^{1}$; exemplary $\gamma(z)$ curves are shown in Fig. 3(c). Several $S_{\gamma}$ measurements were performed experimentally with the same tip for a series of $\gamma_{\text {set }}$ values and three different starting voltage oscillation amplitudes $V_{\mathrm{A}}^{1}$ (start values are increased by $25 \%$ for the $\gamma$-method). Experimental (circles) and simulated (lines) data for $S_{\gamma}$ of these calibration cycles in Fig. 6 display the determined values for $S_{\gamma}$ as a function of $\gamma_{\text {set }}$ and various starting voltage amplitudes $V_{A}^{1}$. The horizontal line marks the true value of $S=S_{\mathrm{FCA}}$ as obtained by the FCA method. As evident from these data, the $\gamma$-method is prone to errors as the result for $S_{\gamma}$ clearly depends on the chosen parameters. Valid results can only be

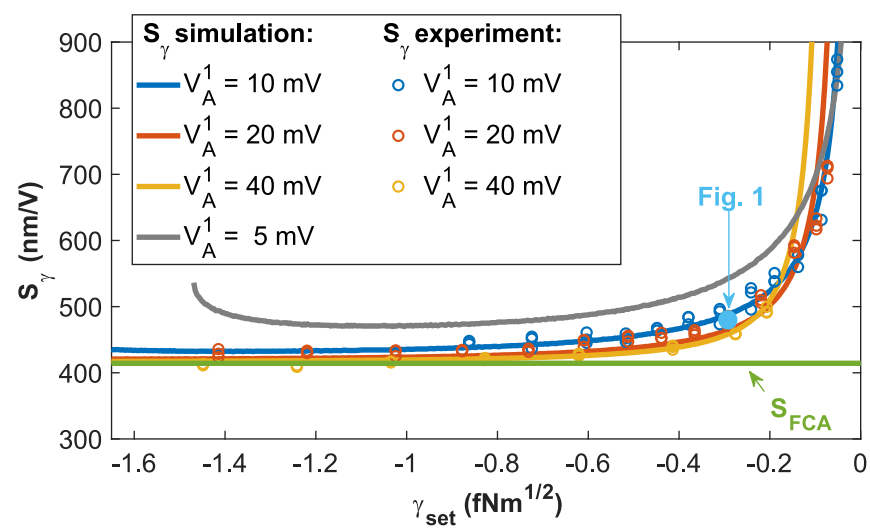

FIG. 6. Simulation and experimental results for determining $S$ using the $\gamma$-method. Experimental data are represented by circles while solid lines are the result of simulations. The value $S_{\gamma}=$ $480 \mathrm{~nm} / \mathrm{V}$ marked by "Fig. 1" is used as the starting value $S_{\gamma}$ for the FCA simulations in Fig. 1. 


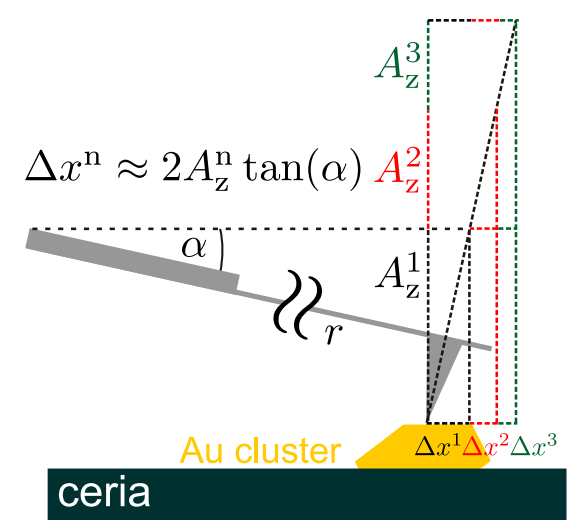

FIG. 7. Effect of the cantilever inclination towards the sample surface. The lateral movement of the tip $\Delta x^{\mathrm{n}}$ from the measurement position above an Au cluster is a function of the oscillation amplitude $A_{\mathrm{z}}^{\mathrm{n}}$.

expected for very large start amplitudes $A$ and in the limit of strong tip-surface interaction $\gamma_{\text {set. }}$. For start amplitudes $A^{1}$ below $5 \mathrm{~nm}$, as are commonly used in NC-AFM imaging, we note that the $S_{\gamma}$ curves do not approach $S_{\mathrm{FCA}}$ but are always offset. In this case, the set point $\gamma_{\text {set }}$ cannot be further reduced beyond about $\gamma_{\text {set }} \sim-1.5 \mathrm{fN} \mathrm{m}^{1 / 2}$ as the minimum of the $\gamma(z)$ curve is already located close to this value. Experimental data in Fig. 6 were taken with a fairly sharp silicon tip interacting with the $\mathrm{CeO}_{2}(111)$ surface. We find that the $S_{\gamma}$ offsets and deviations from $S_{\mathrm{FCA}}$ are even larger when using a tip with a larger tip radius such as a $\mathrm{Pt} / \mathrm{Ir}$-coated coated tip (data not shown). In summary, the $\gamma$-method yields an estimate of $S$ that may be close to the true value, however, it is not a reliable procedure to determine an accurate value for $S$.

\section{APPENDIX C: EFFECTS OF CANTILEVER INCLINATION}

In many experimental setups, the direction of the probe oscillation is inclined by a small angle $\alpha$ with respect to the the surface normal (see Fig. 7). Hence the distinction between two different amplitudes $A$ and $A_{z}$ is important. The first one, $A$, is the physical oscillation amplitude of the cantilever beam end, while $A_{z}$ is the projection on the surface normal vector with $A_{\mathrm{z}}=A \cos \alpha$. The oscillation voltage amplitude $V_{A}$ is measured in the experiment. This voltage amplitude is linked to the physical oscillation amplitude by the sensitivity factor $S=A / V_{\mathrm{A}}$. Hence a sensitivity factor representing the vertical component only can be defined as $S_{\mathrm{z}}=S \cdot \cos \alpha=$ $A_{\mathrm{z}} / V_{\mathrm{A}}$. Consequently, the cantilever inclination not only requires the distinction between two physical amplitudes $A$ and $A_{z}$ but also the distinction between two sensitivity factors $S$ and $S_{z}$.

An inclination of the cantilever oscillation to the surface normal is further relevant when measuring in the vicinity of a small surface feature (see Fig. 7), as it results in a lateral displacement $\Delta x$ between the lower and the upper turning point during every oscillation cycle. Increasing the amplitude $A_{\mathrm{z}}$ results in a larger lateral movement $\Delta x$ of the tip, which can be described by the relation $\Delta x \approx 2 A_{\mathrm{z}} \tan (\alpha)$ (for oscillation amplitudes that are small compared to the cantilever beam length, the circular trajectory can be well approximated by a straight segment). While this lateral movement has no effect on measurements on large, homogeneous, and isotropic areas of the sample surface, it introduces a systematic error when measurements of a small object such as a metal cluster are performed. As Fig. 7 exemplifies, an increase of the amplitude results in a lateral movement of the tip possibly beyond the cluster surface area. Thus, the force gradient in the vicinity of the cluster affect the force measurement, specifically for large oscillation amplitudes. As the force inversion algorithm does not take this into account, but rather considers an oscillation along the surface normal, this results in an erroneous force curve. As seen in Figs. 2(c) and 2(d) of the main paper, this is a notable effect.

\section{APPENDIX D: EXPERIMENTAL METHODS}

Sample preparation and NC-AFM measurements were performed in an ultra-high vacuum (UHV) system at a base pressure in the $10^{-11}$ mbar regime. $\mathrm{CeO}_{2}(111)$ surfaces were prepared on a single crystal (SurfaceNet $\mathrm{GmbH}$, Rheine, Germany) by cycles of argon ion sputtering $\left(p_{A r}=\right.$ $10^{-5} \mathrm{mbar}, U=1.5 \mathrm{kV}, I=15 \mathrm{~mA}, 5 \mathrm{~min}$ ) followed by annealing at high temperature $(1150 \pm 25 \mathrm{~K})$ for 15 minutes, see Refs. [64,65] for further details. Gold was subsequently deposited onto the $\mathrm{CeO}_{2}$ (111) sample held at room temperature by using small gold pieces (Purity: $99.99+\%$,Goodfellow) in a crucible of an electron beam evaporator (type EFM $3 \mathrm{i}$ from Focus GmbH, Huenstetten, Germany). Regularly shaped gold nanoparticles (typical widths of $10 \mathrm{~nm}$ and heights of 1 to $3 \mathrm{~nm}$ ) were formed in a postdeposition annealing step at a temperature of about $(520 \pm 25) \mathrm{K}$.

NC-AFM measurements were conducted using a modified [70] commercial ultra-high vacuum AFM/STM instrument (ScientaOmicron, Taunusstein, Germany) connected to an R9 (RHK Technology, Troy (MI), USA) SPM controller. Additionally, an atom tracking system [53] enabled the measurement and compensation of drift during the experiment. Drift effects were further reduced by a dedicated temperature control of the laboratory. Kelvin probe force microscopy [71] with sideband detection was activated to minimize electrostatic background forces. A bias modulation at a frequency of $f_{\mathrm{el}}=1567 \mathrm{~Hz}$ and with an amplitude of $1 \mathrm{~V}_{p}$ was used. On the gold clusters, a local Kelvin signal up to $V_{\mathrm{KPFM}}=1.7 \mathrm{~V}$ larger compared to the terraces was observed during the measurements presented here.

Experiments were performed with standard silicon (type PPP-NCH, Nanosensors, Switzerland, specified tip radius $<10 \mathrm{~nm}$ ) and platinum iridium (Pt/Ir) coated silicon cantilevers (type NCHPt, Nanosensors, Switzerland, specified tip radius $<25 \mathrm{~nm}$ ). To remove the oxide layer and contaminants, cantilevers were subjected to argon ion sputtering with the following parameters: Si cantilever: $p_{A r}=10^{-5} \mathrm{mbar}, U=$ $0.5 \mathrm{kV}, I=15 \mathrm{~mA}, 5 \mathrm{~min}$; Pt $/ \mathrm{Ir}$-coated cantilever: $p_{A r}=$ $10^{-6}$ mbar, $U=0.5 \mathrm{kV}, I=15 \mathrm{~mA}, 2 \mathrm{~min}$.

A bare silicon and a Pt/Ir-coated silicon cantilever with parameters $f_{0}=277523 \mathrm{~Hz}, k_{0}=57.43 \mathrm{~N} / \mathrm{m}$, and $Q=22000$ (bare silicon) as well as $f_{0}=277203 \mathrm{~Hz}, k_{0}=18.58 \mathrm{~N} / \mathrm{m}$, and $Q=18900(\mathrm{Pt} / \mathrm{Ir}$-coated silicon) were used during the experiments. The cantilever-specific force constants were measured using the thermal method [67] and the eigenfre- 


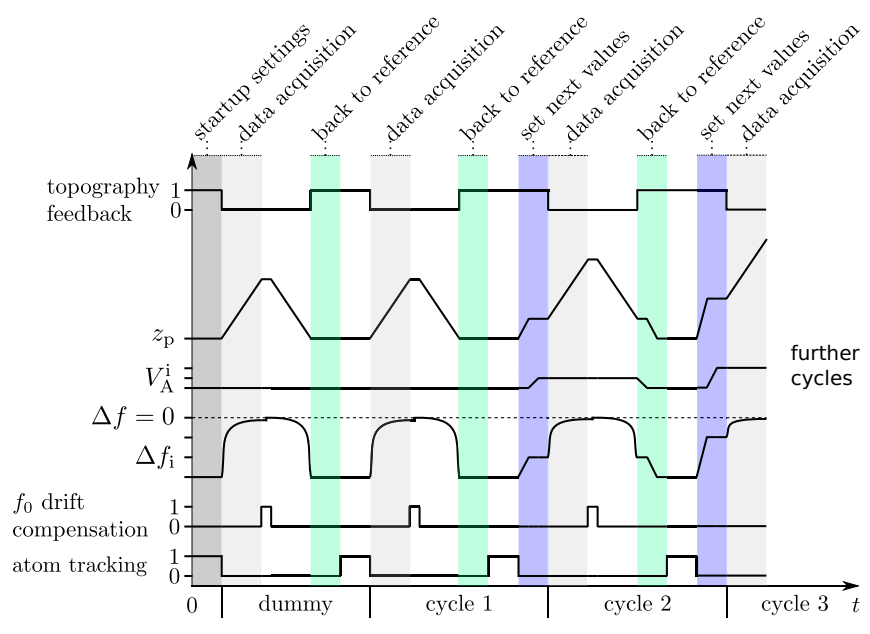

FIG. 8. Data acquisition protocol for FCA.

quencies determined with the tip retracted by about $60 \mathrm{~nm}$ from the surface. Due to the system geometry, the direction of the cantilever oscillation is tilted with respect to the surface normal by $\alpha \approx 12.5^{\circ}$.

\section{APPENDIX E: FCA MEASUREMENT PROTOCOL}

A set of $2 N$ distance-dependent $\Delta f\left(z_{\mathrm{p}}\right)$ curves is acquired with ascending and descending voltage amplitude values $V_{A}^{i}\left(V_{A}^{1}, V_{A}^{2}, \ldots, V_{A}^{N}, V_{A}^{N}, V_{A}^{N-1}, \ldots, V_{A}^{1}\right)$.

Data acquisition is started when sufficient time has passed after the initial approach to reduce piezo creep artefacts. At this point, atom tracking is activated to measure the residual drift and to compensate for the linear component by the feedforward technique [53]. It is helpful to acquire a "dummy" curve before the first measurement curve to ensure that the piezo scanner yields a similar response during the whole measurement set.

An initial estimate for the sensitivity factor $S$ is made using the $\gamma$-method. Using this estimate, a series $V_{A}^{1}, \ldots, V_{A}^{N}$ of $N$ voltage amplitudes is chosen in the physical amplitude range of interest. For the first voltage amplitude $V_{A}^{1}$ and a suitable frequency shift setpoint $\Delta f_{1}$, the unscaled normalised frequency shift $\gamma^{\star}=\gamma_{1} \cdot f_{0} /\left(k_{0} S^{3 / 2}\right)=\left(V_{A}^{1}\right)^{3 / 2} \Delta f_{1}$ is calculated. The data acquisition procedure for each amplitude value proceeds along the steps schematically depicted in Fig. 8. These steps are repeated $2 N$ times for $N$ different amplitudes using ascending and descending voltage amplitude values.

(1) Atom tracking is used to measure lateral and vertical drift. Based on this measurement, the linear drift vector for the feed-forward compensation is updated, feed-forward activated, and atom-tracking stopped.

(2) The voltage amplitude is set to the next value and the frequency-shift set point is adjusted according to $\gamma^{\star}$. To reduce the risk of tip instabilities, the frequency shift setpoint is increased after increasing the amplitude value and decreased before decreasing the amplitudes value.

(3) The topography feedback is switched off.

(4) The NC-AFM signals are sampled while ramping $z_{\mathrm{p}}$ through the given range. The $z_{\mathrm{p}}$ ramp range has to be chosen to be at least $2 A_{\max }$ for the largest amplitude $A_{\max }$ for a reliable

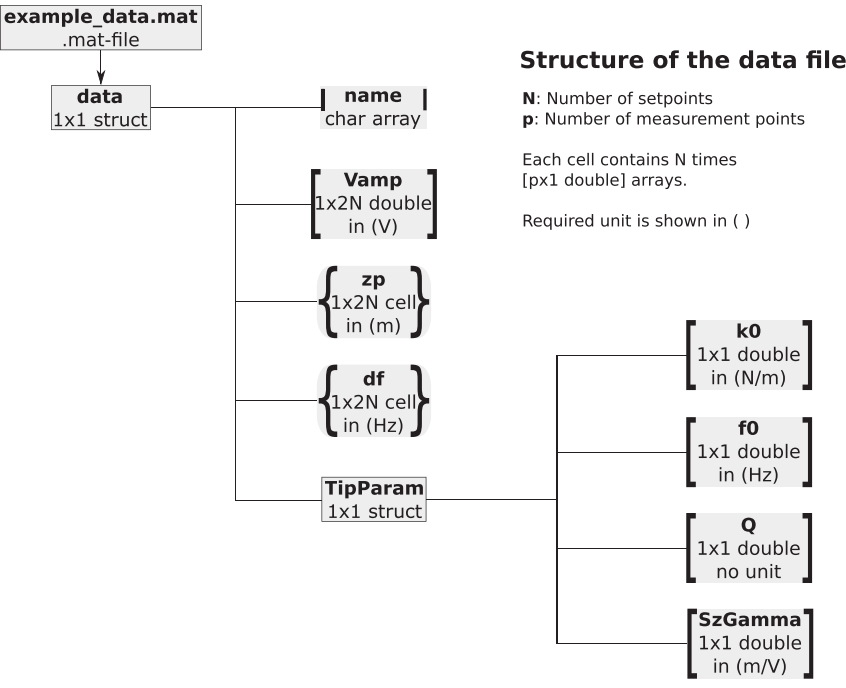

FIG. 9. Schematic construction of the struct EXAMPLE_DATA.MAT in MATLAB.

force calculation. In the present case, we choose amplitudes up to about $20 \mathrm{~nm}$ with a $z_{\mathrm{p}}$ range of $60 \mathrm{~nm}$ in $25 \mathrm{pm}$ steps.

(5) With the tip held at the largest separation to the surface, the center frequency $f_{0}$ is readjusted in the phase-locked loop to compensate for thermal drift of the center frequency.

(6) The tip is then moved back to the surface and the topography feedback is reactivated.

(7) The procedure is repeated by starting with step 1 until all voltage amplitude values are processed.

We typically measure a total of 12 or $14 \Delta f(z)$ curves per position, thus using six or seven different amplitudes. To determine nonlinear drift and creep artifacts in the dataset, it is most helpful to acquire the curves in equidistant time steps. Hence, the complete FCA measurement protocol is implemented in a script form to ensure that the curves are measured in equidistant time steps $t_{\text {cycle }}$.

\section{APPENDIX F: FCA DATA ANALYSIS PROCEDURE}

The true force-distance curve is derived from the measured set of $\Delta f\left(z_{\mathrm{p}}\right)$ curves by a multiparameter optimization algorithm. This algorithm is implemented in the MATLAB method applyFCA explained here in detail. The applyFCA function requires as input several arguments for the optimisation process. These are SGFILTPARAM, NODEPARAM, OPTIONS, DATA_PATH, and SAVE_PATH. SGFILTPARAM is a MATLAB struct containing information about the frame size and order of the Savitzky-Golay filter [72] applied during the force inversion for calculating the derivative [73]. All information about the node placement on the reference force curve is saved in the struct NODEPARAM. The OPTIONS struct contains the options for the built-in MATLAB function FMINSEARCH utilized by this implementation. Default values suitable for FCA are included. The results of APPLYFCA are saved in the directory set in SAVE_PATH and the data file is loaded from the location given in DATA_PATH. By running the script RUN_FCA.M, all parameters are set and inserted into the function APPLYFCA. The complete optimization can directly 


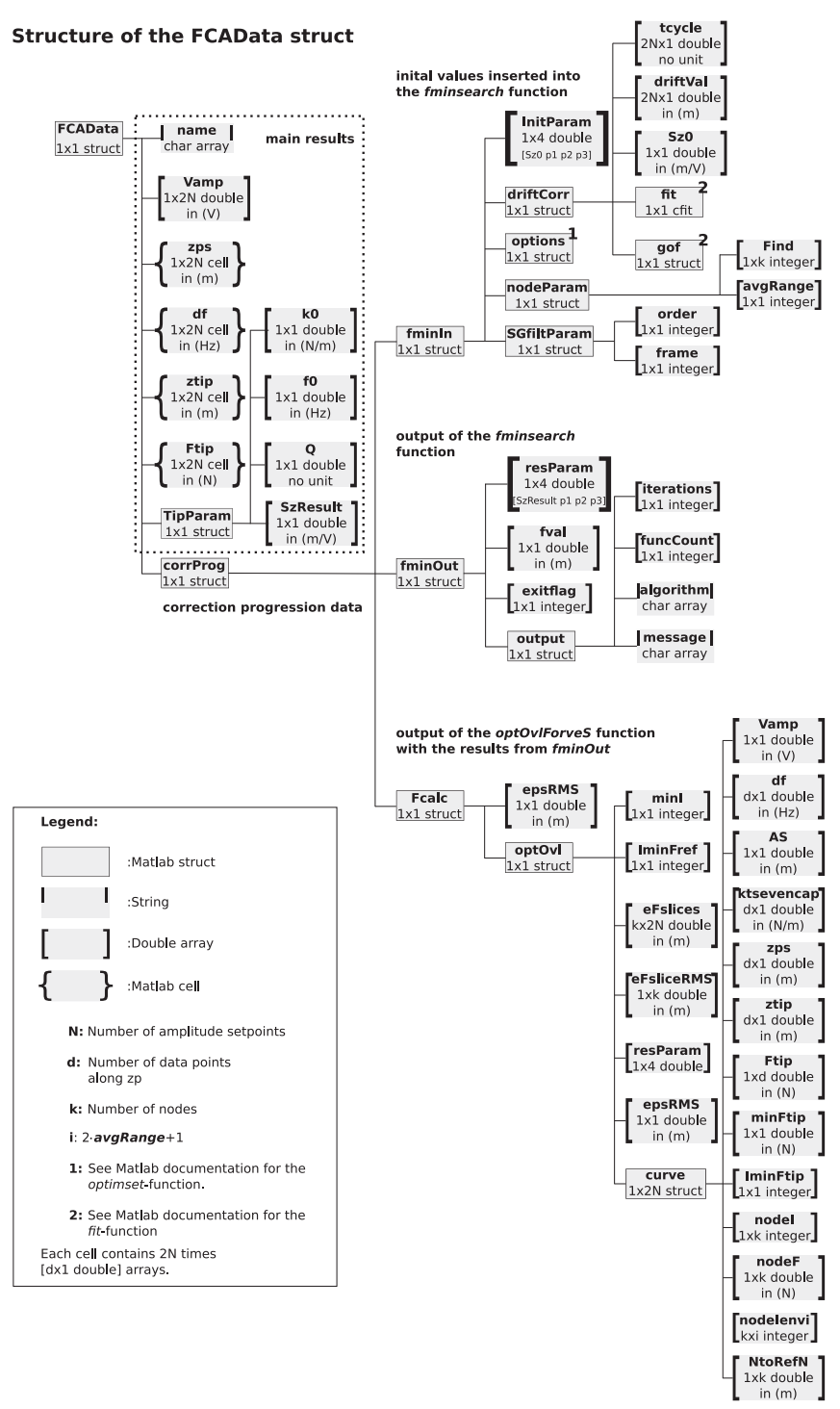

FIG. 10. Schematic overview of the output data of FCA.

be run by using the example data provided in the file EXAMPLE_DATA.MAT (see Fig. 9). The corresponding results (structure of the result struct shown in Fig. 10) are stored in the file EXAMPLE_DATA_FCARESULT.MAT. This file resides in the SAVE_PATH folder as the input data after running RUN_FCA.M. The input data file (here EXAMPLE_DATA.MAT) is copied to the output folder and thus stored with all generated output data. The example data fulfill the minimum requirements of one measured data set as outlined in Fig. 9. The minimum requirements are matrices with the frequency shift data (field $d f$ ), the voltage amplitudes (field Vamp), the piezopositions (field $z p$ ), the basic cantilevers properties determined in the experiment (force constant $k 0$, cantilever eigenfrequency $f 0$, and quality factor $Q$ ), and an estimation of the sensitivity factor $S_{z}$ which is here stored in the field $S z$ Guess.

The flowchart in Fig. 11 depicts the work flow of the FCA method as implemented in the script RUN_FCA.M. This script executes the optimization process in APPLYFCA as the central routine. In a first step, initial parameters for $S_{z}$ and for the drift compensation are calculated. With an initial estimation

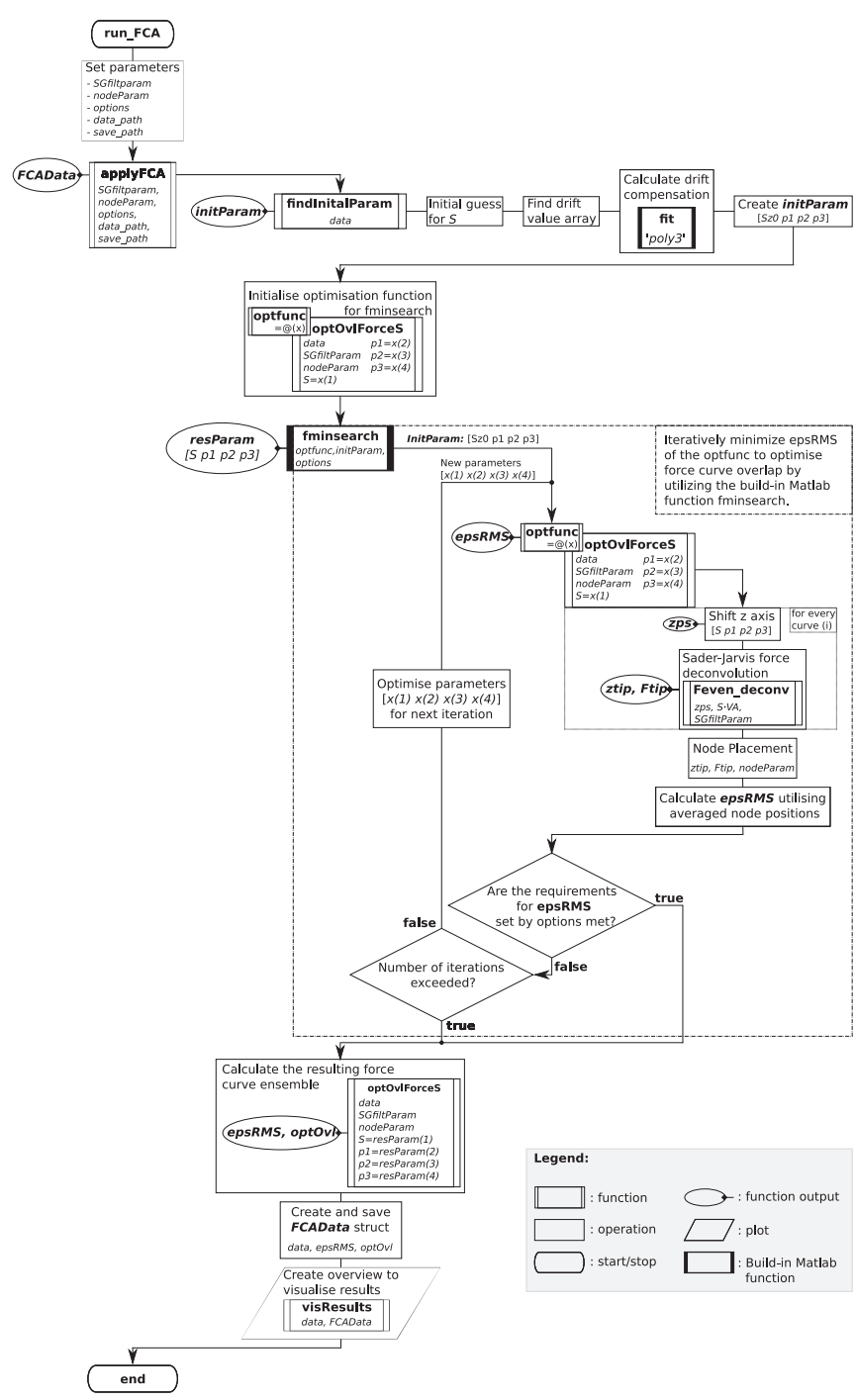

FIG. 11. Flowchart for FCA analysis procedure.

for $S_{z}$ (e.g., by using $S_{\gamma, z}$ ), the voltage amplitudes $V_{A}^{i}$ are converted to the physical oscillation amplitudes $A^{i}$. The drift is approximated by a third-order polynomial. The initial $S_{z}$ value and the polynomial coefficients $p 1, p 2, p 3$ are saved in INITPARAM. This four parameter tuple $\left(S_{z}, p 1, p 2, p 3\right)$ is optimized to find a minimum deviation between nodes placed on the set of force curves utilising the build-in MATLAB function FMINSEARCH. The force conversion is executed by the script FEVEN_DECONV.M using the inversion algorithm by Sader and Jarvis [48]. In the set of force curves, six nodes with equivalent forces are placed within the region of steep slope of every force curve. To prohibit that the nodes are affected by data outliers, every node is averaged over five samples. As a measure of the curve's deviation, the RMS deviation (epsRMS) of all nodes with respect to those of a reference curve is calculated. Hence, the final EPSRMS value is a measure for the quality of the optimisation. The reference curve is the first curve of the measurement set, it is drift free and thus is not affected by the drift correction but rather only shifted by $S_{z}$. The nodes are placed with respect to the smallest shared absolute force value of the complete force curve ensemble. This optimization 

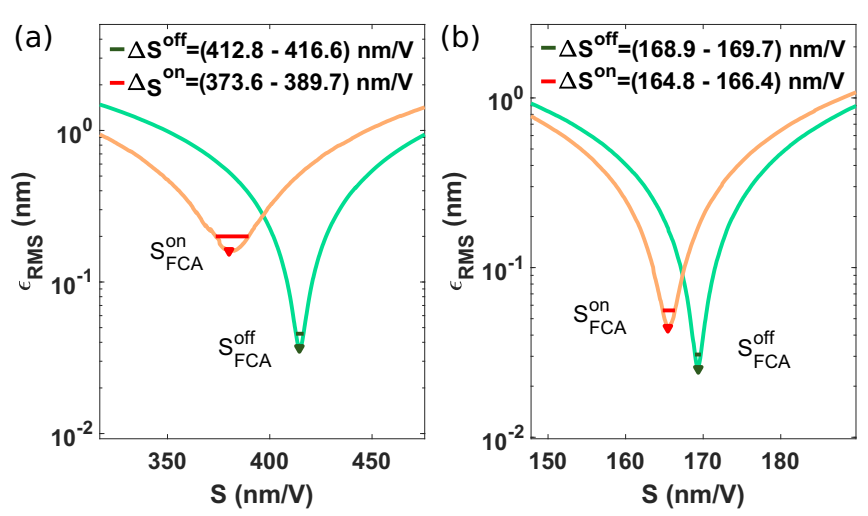

FIG. 12. Error analysis for the FCA method. The width of the $\epsilon_{\mathrm{RMS}}(S)$ curves $25 \%$ above the minimum $\epsilon_{\mathrm{RMS}}$ value is marked by horizontal lines. Analysis for (a) silicon and (b) Pt/Ir-coated tip.

routine terminates when a specific threshold value for the optimization or an iteration limit is reached. This termination criteria can be set in the OPTIONS struct for FMINSEARCH. Note that the code strictly uses $S_{z}$, the conversion to $S$ has to be performed with the resulting value.

\section{APPENDIX G: ERROR ANALYSIS}

To quantify the quality of the FCA optimization, we introduce an analysis based on the $\epsilon_{\mathrm{RMS}}(S)$ curves as shown in Fig. 12. The curves are the result of keeping the optimized drift polynomial constant and calculating $\epsilon_{\mathrm{RMS}}$ for $S$ values around the minimal value $S_{\mathrm{FCA}}$. The width of the curves around the minimum can be interpreted as the uncertainty of the optimized result due to experimental errors where a narrow dip in $\epsilon_{\mathrm{RMS}}(S)$ reflects a small uncertainty in the optimized force curves. As a conservative estimate for the accuracy of $S_{\mathrm{FCA}}$, we determine the full width of the $\epsilon_{\mathrm{RMS}}(S)$ curve at an $\epsilon_{\mathrm{RMS}}$ value $25 \%$ above the minimum value. This is shown for the measurements with the silicon [Fig. 12(a)] and Pt/Ir coated cantilever [Fig. 12(b)] and yields $S_{\mathrm{FCA}}^{\text {off }}=(414.6 \pm 1.9) \mathrm{nm} / \mathrm{V}$ and $S_{\mathrm{FCA}}^{\text {on }}=(383 \pm$ $8) \mathrm{nm} / \mathrm{V}$ for the bare silicon tip and $S_{\mathrm{FCA}}^{\text {off }}=(169.3 \pm$ $0.5) \mathrm{nm} / \mathrm{V}$ and $S_{\mathrm{FCA}}^{\mathrm{on}}=(165.5 \pm 0.9) \mathrm{nm} / \mathrm{V}$ for the $\mathrm{Pt} / \mathrm{Ir}-$ coated tip. For both off cluster measurements, $S$ varies by less than $0.5 \%$, highlighting the robustness of the FCA method.

\section{APPENDIX H: FCA WITH MATRIX FORCE INVERSION ALGORITHM}

All results in the main paper are based on the FCA method utilizing the force inversion algorithm introduced by Sader and Jarvis [48]. In this Appendix we demonstrate that FCA can be performed equally well with the MATRIX algorithm [47] for force inversion with the comparison reproducing known differences between the two algorithms [50].

Figure 13 presents results of FCA with the MATRIX algorithm applied to the datasets from the simulation [Fig. 13(a)] as well as from the experimental off-cluster measurements with the silicon [Fig. 13(b)] and Pt/Ir-coated [Fig. 13(c)] tips. The input data for FCA with the MATRIX algorithm used in Fig. 13 are identical to the data used in the main paper for FCA with the Sader-Jarvis algorithm (see Figs. 1 and 2 of the main paper) besides applying a Savitzky-Golay smoothing filter [72] with frame size 22 and of order 2 to the frequency shift $\Delta f$ data. We chose to apply this prefilter as a strategy to yield similar noise levels in the resulting force data as achieved with FCA+Sader/Jarvis. In the latter case, a Savitzky-Golay filter is used at the derivation step (see also Appendix F), however, a similar strategy for calculating the derivative in the MATRIX algorithm is not provided. Thus, we revert to filtering the input $\Delta f$ data.

The results for the sensitivity factor $S$ are virtually identical to the ones yielded with the Sader-Jarvis algorithm. The force curves resulting from the simulated data [Fig. 13(a)] show a perfect overlap and the residual error $\epsilon_{\mathrm{RMS}}$ represents again solely the numerical error. An excellent overlap of the force
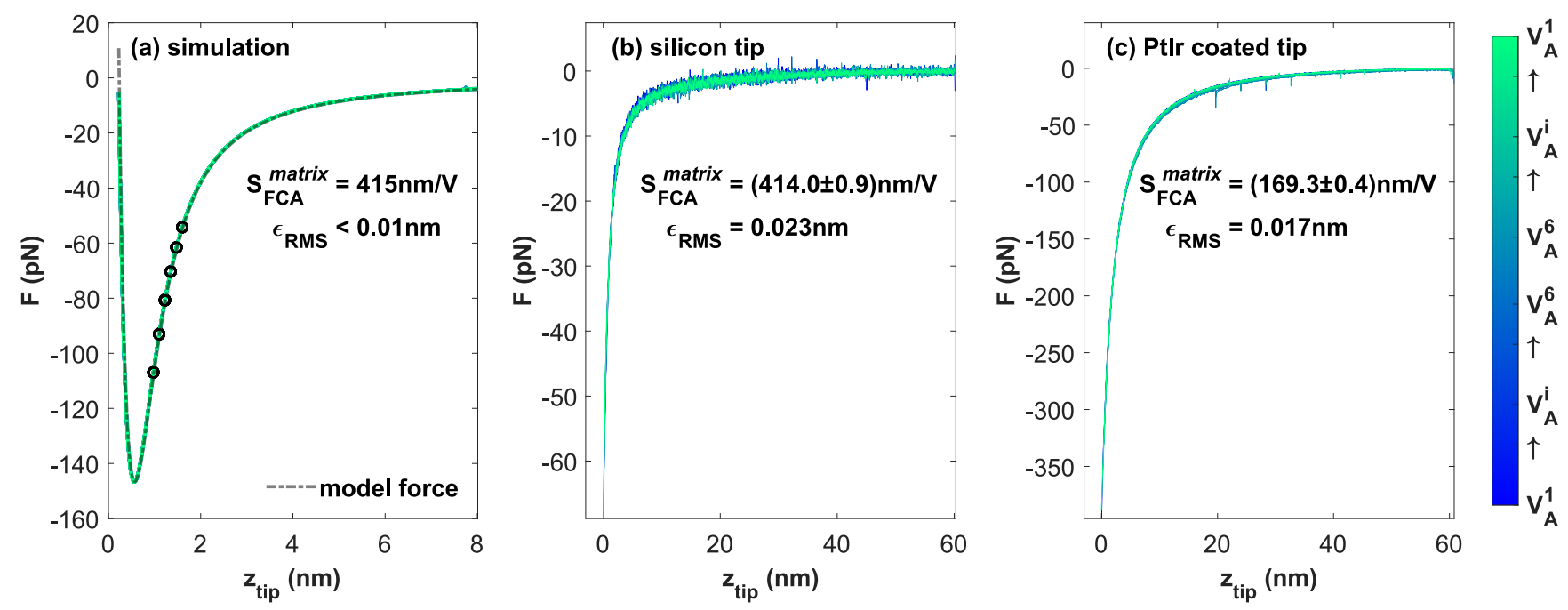

FIG. 13. Results of the FCA method utilising the MATRIX force inversion algorithm [47] for (a) a simulated data set (identical model force as in the main paper, see also Fig. 1) and experimental measurements performed with (b) a silicon tip [off-cluster, see also Fig. 2(c)], and (c) a Pt/Ir-coated tip [off-cluster, see also Fig. 2(d)]. 
curves with slightly smaller $\epsilon_{\mathrm{RMS}}$ values are also found for the experimental data acquired with the silicon and Pt/Ir-coated tips. The slight differences in the resulting values, in the noise levels, and the number of single outliers apparent in Figs. 13(b) and 13(c) are explained by the different filtering strategies as outlined before. We found that the sensitivity values $S$ calculated with FCA+MATRIX slightly differ at decimal places when modifying the filter parameters, yet, these fluctuations are well within the accuracy of the FCA method. A detailed discussion on the differences between the two force inversion algorithms has been presented before by Welker et al. [50].
[1] L. Kantorovich, Quantum Theory of the Solid State: An Introduction, Fundamental Theories of Physics, Vol. 136 (Springer Science, Dordrecht, The Netherlands, 2004), p. 626.

[2] J. N. Israelachvili, Intermolecular and Surface Forces (Third Edition) (Academic, San Diego, CA, 2011), p. iii.

[3] H. J. Mamin and D. Rugar, Appl. Phys. Lett. 79, 3358 (2001).

[4] L. L. Liu, S. Kheifets, V. Ginis, and F. Capasso, Phys. Rev. Lett. 116, 228001 (2016).

[5] V. Blums, M. Piotrowski, M. I. Hussain, B. G. Norton, S. C. Connell, S. Gensemer, M. Lobino, and E. W. Streed, Sci. Adv. 4, eaao4453 (2018).

[6] M. A. Lantz, H. J. Hug, R. Hoffmann, P. J. A. van Schendel, P. Kappenberger, S. Martin, A. Baratoff, and H. J. Güntherodt, Science 291, 2580 (2001).

[7] Y. Sugimoto, P. Pou, M. Abe, P. Jelinek, R. Pérez, S. Morita, and O. Custance, Nature 446, 64 (2007).

[8] H. F. Wen, Q. Z. Zhang, Y. Adachi, M. Miyazaki, Y. Sugawara, and Y. J. Li, Appl. Surf. Sci. 505, 144623 (2020).

[9] A. M. Sweetman, S. P. Jarvis, H. Q. Sang, I. Lekkas, P. Rahe, Y. Wang, J. B. Wang, N. R. Champness, L. Kantorovich, and P. Moriarty, Nat. Commun. 5, 3931 (2014).

[10] P. Jelínek, J. Phys.: Condens. Matter 29, 343002 (2017).

[11] H. Mönig, S. Amirjalayer, A. Timmer, Z. Hu, L. Liu, O. Díaz Arado, M. Cnudde, C. A. Strassert, W. Ji, M. Rohlfing, and H. Fuchs, Nat. Nanotechnol. 13, 371 (2018).

[12] L. Costa, M. S. Rodrigues, E. Newman, C. Zubieta, J. Chevrier, and F. Comin, J. Mol. Recognit. 26, 689 (2013).

[13] S. Senapati, A. B. Poma, M. Cieplak, S. Filipek, and P. S. H. Park, Anal. Chem. 91, 7226 (2019).

[14] F. S. Ruggeri, T. Sneideris, M. Vendruscolo, and T. P. J. Knowles, Arch. Biochem. Biophys. 664, 134 (2019).

[15] E. T. Herruzo, H. Asakawa, T. Fukuma, and R. Garcia, Nanoscale 5, 2678 (2013).

[16] E. I. Altman, M. Z. Baykara, and U. D. Schwarz, Acc. Chem. Res. 48, 2640 (2015).

[17] A. Sweetman, M. Rashid, S. Jarvis, J. Dunn, P. Rahe, and P. Moriarty, Nat. Commun. 7, 10621 (2016).

[18] D. Martin-Jimenez and R. Garcia, J. Phys. Chem. Lett. 8, 5707 (2017).

[19] J. Laurent, H. Sellier, A. Mosset, S. Huant, and J. Chevrier, Phys. Rev. B 85, 035426 (2012).

[20] E. Voloshina and Y. Dedkov, Phys. Rev. B 93, 235418 (2016).

[21] L. A. Jurado, H. Kim, A. Rossi, A. Arcifa, J. K. Schuh, N. D. Spencer, C. Leal, R. H. Ewoldt, and R. M. Espinosa-Marzal, Phys. Chem. Chem. Phys. 18, 22719 (2016).

[22] L. R. Dickinson, B. M. J. M. Suijkerbuijk, S. Berg, F. H. M. Marcelis, and H. C. Schniepp, Energy \& Fuels 30, 9193 (2016).

[23] P. M. Claesson, I. Dobryden, G. Li, Y. J. He, H. Huang, P. A. Thoren, and D. B. Haviland, Phys. Chem. Chem. Phys. 19, 23642 (2017).
[24] R. Pawlak, C. Drechsel, P. D’Astolfo, M. Kisiel, E. Meyer, and J. I. Cerda, Proc. Nat. Acad. Sci. USA 117, 228 (2020).

[25] C. Chiutu, A. M. Sweetman, A. J. Lakin, A. Stannard, S. Jarvis, L. Kantorovich, J. L. Dunn, and P. Moriarty, Phys. Rev. Lett. 108, 268302 (2012).

[26] M. S. Rodrigues, L. Costa, J. Chevrier, and F. Comin, Appl. Phys. Lett. 101, 203105 (2012).

[27] A. Caló, O. V. Robles, S. Santos, and A. Verdaguer, Beilstein J. Nanotechnol. 6, 809 (2015).

[28] S. Kawai, A. S. Foster, T. Björkman, S. Nowakowska, J. Björk, F. F. Canova, L. H. Gade, T. A. Jung, and E. Meyer, Nat. Commun. 7, 11559 (2016).

[29] M. J. Shon, S. H. Rah, and T. Y. Yoon, Sci. Adv. 5, 12 (2019).

[30] T. Fukuma, B. Reischl, N. Kobayashi, P. Spijker, F. F. Canova, K. Miyazawa, and A. S. Foster, Phys. Rev. B 92, 155412 (2015).

[31] H. Söngen, B. Reischl, K. Miyata, R. Bechstein, P. Raiteri, A. L. Rohl, J. D. Gale, T. Fukuma, and A. Kühnle, Phys. Rev. Lett. 120, 116101 (2018).

[32] H. Söngen, Y. M. Morais Jaques, L. Zivanovic, S. Seibert, R. Bechstein, P. Spijker, H. Onishi, A. S. Foster, and A. Kühnle, Phys. Rev. B 100, 205410 (2019).

[33] B. Reischl, P. Raiteri, J. D. Gale, and A. L. Rohl, J. Phys. Chem. C 123, 14985 (2019).

[34] A. Sweetman, J. Stirling, S. P. Jarvis, P. Rahe, and P. Moriarty, Phys. Rev. B 94, 115440 (2016).

[35] J. Onoda, M. Ondráček, P. Jelínek, and Y. Sugimoto, Nat. Commun. 8, 15155 (2017).

[36] J. N. Ladenthin, T. Frederiksen, M. Persson, J. C. Sharp, S. Gawinkowski, J. Waluk, and T. Kumagai, Nat. Chem. 8, 935 (2016).

[37] S. Garcia-Manyes and A. E. M. Beedle, Nat. Rev. Chem. 1, 0083 (2017).

[38] A. Sweetman, I. Lekkas, and P. Moriarty, J. Phys.: Condens. Matter 29, 074003 (2017).

[39] C. Wagner, M. F. B. Green, P. Leinen, T. Deilmann, P. Krüger, M. Rohlfing, R. Temirov, and F. S. Tautz, Phys. Rev. Lett. 115, 026101 (2015).

[40] D. Rugar, R. Budakian, H. J. Mamin, and B. W. Chui, Nature 430, 329 (2004).

[41] L. Thiel, Z. Wang, M. A. Tschudin, D. Rohner, I. GutiérrezLezama, N. Ubrig, M. Gibertini, E. Giannini, A. F. Morpurgo, and P. Maletinsky, Science 364, 973 (2019).

[42] Z. Sun, M. P. Boneschanscher, I. Swart, D. Vanmaekelbergh, and P. Liljeroth, Phys. Rev. Lett. 106, 046104 (2011).

[43] C. Barth, A. S. Foster, C. R. Henry, and A. L. Shluger, Adv. Mater. 23, 477 (2011).

[44] H. Söngen, R. Bechstein, and A. Kühnle, J. Phys.: Condens. Matter 29, 274001 (2017).

[45] B. Voigtländer, Atomic Force Microscopy, 2nd ed., NanoScience and Technology (Springer Nature, Cham, Switzerland, 2019). 
[46] M. Guggisberg, M. Bammerlin, C. Loppacher, O. Pfeiffer, A. Abdurixit, V. Barwich, R. Bennewitz, A. Baratoff, E. Meyer, and H. J. Güntherodt, Phys. Rev. B 61, 11151 (2000).

[47] F. J. Giessibl, Appl. Phys. Lett. 78, 123 (2001).

[48] J. E. Sader and S. P. Jarvis, Appl. Phys. Lett. 84, 1801 (2004).

[49] J. E. Sader, B. D. Hughes, F. Huber, and F. J. Giessibl, Nat. Nanotechnol. 13, 1088 (2018).

[50] J. Welker, E. Illek, and F. J. Giessibl, Beilstein J. Nanotechnol. 3, 238 (2012).

[51] J. Lübbe, M. Temmen, P. Rahe, and M. Reichling, Beilstein J. Nanotechnol. 7, 1885 (2016).

[52] M. Abe, Y. Sugimoto, T. Namikawa, K. Morita, N. Oyabu, and S. Morita, Appl. Phys. Lett. 90, 203103 (2007).

[53] P. Rahe, J. Schütte, W. Schniederberend, M. Reichling, M. Abe, Y. Sugimoto, and A. Kühnle, Rev. Sci. Instrum. 82, 063704 (2011).

[54] J. F. Portolés, P. J. Cumpson, J. Hedley, S. Allen, P. M. Williams, and S. J. B. Tendler, J. Exp. Nanosci. 1, 51 (2006).

[55] G. H. Simon, M. Heyde, and H.-P. Rust, Nanotechnology 18, 255503 (2007).

[56] Y. Sugimoto, Y. Nakajima, D. Sawada, K. I. Morita, M. Abe, and S. Morita, Phys. Rev. B 81, 245322 (2010).

[57] J. F. Gonzales Martinez, I. Nieto-Carvajal, and J. Colchero, Nanotechnology 24, 185701 (2013).

[58] S. S. Borysov, D. Forchheimer, and D. B. Haviland, Beilstein J. Nanotechnol. 5, 1899 (2014).

[59] F. J. Azcona, A. Jha, C. Yáñez, R. Atashkhooei, and S. Royo, Sensors 16, 997 (2016).
[60] L. Hao, Q. Wang, P. Peng, Z. Cao, W. Jiao, F. Yang, W. Liu, R. Wang, and X. He, Ultramicroscopy 174, 106 (2017).

[61] O. E. Dagdeviren, Y. Miyahara, A. Mascaro, and P. Grütter, Rev. Sci. Instrum. 90, 013703 (2019).

[62] F. J. Giessibl, Phys. Rev. B 56, 16010 (1997).

[63] See Supplemental Material at http://link.aps.org/supplemental/ 10.1103/PhysRevB.103.075409 for the program code.

[64] H. H. Pieper, C. Lammers, L. Tröger, S. Bahr, and M. Reichling, Rev. Sci. Instrum. 83, 055110 (2012).

[65] H. H. Pieper, C. Derks, M. H. Zoellner, R. Olbrich, L. Tröger, T. Schroeder, M. Neumann, and M. Reichling, Phys. Chem. Chem. Phys. 14, 15361 (2012).

[66] R. Olbrich, G. E. Murgida, V. Ferrari, C. Barth, A. M. Llois, M. Reichling, and M. V. Ganduglia-Pirovano, J. Phys. Chem. C 121, 6844 (2017).

[67] J. Lübbe, M. Temmen, P. Rahe, A. Kühnle, and M. Reichling, Beilstein J. Nanotechnol. 4, 227 (2013).

[68] S. Kuhn and P. Rahe, Phys. Rev. B 89, 235417 (2014).

[69] C. Argento and R. H. French, J. Appl. Phys. 80, 6081 (1996).

[70] S. Torbrügge, J. Lübbe, L. Tröger, M. Cranney, T. Eguchi, Y. Hasegawa, and M. Reichling, Rev. Sci. Instrum. 79, 083701 (2008).

[71] M. Nonnenmacher, M. P. O’Boyle, and H. K. Wickramasinghe, Appl. Phys. Lett. 58, 2921 (1991).

[72] A. Savitzky and M. J. E. Golay, Anal. Chem. 36, 1627 (1964).

[73] S. Kuhn, M. Kittelmann, Y. Sugimoto, M. Abe, A. Kühnle, and P. Rahe, Phys. Rev. B 90, 195405 (2014). 